\title{
\begin{tabular}{l|l} 
MitTraries & DSpace@MIT
\end{tabular}
}

\author{
MIT Open Access Articles
}

\section{NUSTAR DISCOVERY OF A 3.76 s TRANSIENT MAGNETAR NEAR SAGITTARIUS A*}

The MIT Faculty has made this article openly available. Please share how this access benefits you. Your story matters.

Citation: Mori, Kaya, Eric V. Gotthelf, Shuo Zhang, Hongjun An, Frederick K. Baganoff, Nicolas M. Barriere, Andrei M. Beloborodov, et al. “NuSTAR DISCOVERY OF A 3.76 s TRANSIENT MAGNETAR NEAR SAGITTARIUS A*." The Astrophysical Journal 770, no. 2 (June 20, 2013): L23. (C) 2013 The American Astronomical Society

As Published: http://dx.doi.org/10.1088/2041-8205/770/2/L23

Publisher: IOP Publishing

Persistent URL: http://hdl.handle.net/1721.1/93747

Version: Final published version: final published article, as it appeared in a journal, conference proceedings, or other formally published context

Terms of Use: Article is made available in accordance with the publisher's policy and may be subject to US copyright law. Please refer to the publisher's site for terms of use. 


\title{
NuSTAR DISCOVERY OF A 3.76 s TRANSIENT MAGNETAR NEAR SAGITTARIUS A*
}

\author{
Kaya Mori ${ }^{1}$, Eric V. Gotthelf ${ }^{1}$, Shuo Zhang ${ }^{1}$, Hongunn An ${ }^{2}$, Frederick K. BaganofF ${ }^{3}$, Nicolas M. Barrière ${ }^{4}$, \\ Andrei M. Beloborodov ${ }^{1}$, Steven E. Boggs ${ }^{4}$, Finn E. Christensen ${ }^{5}$, William W. Craig ${ }^{4}$, Francois Dufour $^{2}$, \\ Brian W. Grefenstette ${ }^{6}$, Charles J. Hailey ${ }^{1}$, Fiona A. Harrison ${ }^{6}$, Jaesub Hong ${ }^{7}$, Victoria M. Kaspi ${ }^{2}$, \\ Jamie A. Kennea ${ }^{8}$, Kristin K. Madsen ${ }^{6}$, Craig B. Markwardt ${ }^{9}$, Melania Nynka ${ }^{1}$, Daniel Stern $^{10}$, \\ JOHN A. TOMSICK ${ }^{4}$, AND WILliam W. ZhaNG ${ }^{9}$ \\ ${ }^{1}$ Columbia Astrophysics Laboratory, Columbia University, New York, NY 10027, USA; kaya@astro.columbia.edu \\ 2 Department of Physics, McGill University, Montreal, QC H3A 2T8, Canada \\ ${ }^{3}$ Kavli Institute for Astrophysics and Space Research, Massachusetts Institute of Technology, Cambridge, MA 02139, USA \\ ${ }^{4}$ Space Sciences Laboratory, University of California, Berkeley, CA 94720, USA \\ ${ }^{5}$ DTU Space-National Space Institute, Technical University of Denmark, Elektrovej 327, 2800 Lyngby, Denmark \\ ${ }^{6}$ Cahill Center for Astronomy and Astrophysics, California Institute of Technology, Pasadena, CA 91125, USA \\ ${ }^{7}$ Harvard-Smithsonian Center for Astrophysics, Cambridge, MA 02138, USA \\ ${ }^{8}$ Department of Astronomy and Astrophysics, The Pennsylvania State University, University Park, PA 16802, USA \\ ${ }^{9}$ NASA Goddard Space Flight Center, Greenbelt, MD 20771, USA \\ ${ }^{10}$ Jet Propulsion Laboratory, California Institute of Technology, Pasadena, CA 91109, USA \\ Received 2013 May 7; accepted 2013 May 14; published 2013 May 30
}

\begin{abstract}
We report the discovery of $3.76 \mathrm{~s}$ pulsations from a new burst source near Sgr A* observed by the NuSTAR observatory. The strong signal from SGR J1745-29 presents a complex pulse profile modulated with pulsed fraction $27 \% \pm 3 \%$ in the $3-10 \mathrm{keV}$ band. Two observations spaced nine days apart yield a spin-down rate of $\dot{P}=(6.5 \pm 1.4) \times 10^{-12}$. This implies a magnetic field $B=1.6 \times 10^{14} \mathrm{G}$, spin-down power $\dot{E}=5 \times 10^{33} \mathrm{erg} \mathrm{s}^{-1}$, and characteristic age $P / 2 \dot{P}=9 \times 10^{3}$ yr for the rotating dipole model. However, the current $\dot{P}$ may be erratic, especially during outburst. The flux and modulation remained steady during the observations and the 3-79 keV spectrum is well fitted by a combined blackbody plus power-law model with temperature $k T_{\mathrm{BB}}=0.96 \pm 0.02 \mathrm{keV}$ and photon index $\Gamma=1.5 \pm 0.4$. The neutral hydrogen column density $\left(N_{\mathrm{H}} \sim 1.4 \times 10^{23} \mathrm{~cm}^{-2}\right)$ measured by NuSTAR and Swift suggests that SGR J1745-29 is located at or near the Galactic center. The lack of an X-ray counterpart in the published Chandra survey catalog sets a quiescent $2-8 \mathrm{keV}$ luminosity limit of $L_{x} \lesssim 10^{32} \mathrm{erg} \mathrm{s}^{-1}$. The bursting, timing, and spectral properties indicate a transient magnetar undergoing an outburst with $2-79 \mathrm{keV}$ luminosity up to $3.5 \times 10^{35} \mathrm{erg} \mathrm{s}^{-1}$ for a distance of $8 \mathrm{kpc}$. SGR J1745-29 joins a growing subclass of transient magnetars, indicating that many magnetars in quiescence remain undetected in the X-ray band or have been detected as high-B radio pulsars. The peculiar location of SGR J1745-29 has important implications for the formation and dynamics of neutron stars in the Galactic center region.
\end{abstract}

Key words: Galaxy: center - pulsars: general - pulsars: individual (SGR J1745-29) - stars: neutron

Online-only material: color figure

\section{INTRODUCTION}

The small class of young neutron stars that exhibit sudden bright X-ray and soft gamma-ray bursts, large X-ray flares, and often strong, broad X-ray pulsations are believed to be "magnetars": neutron stars whose radiation is powered by the decay of intense magnetic fields (Thompson \& Duncan 1995, 1996; Thompson et al. 2002; Beloborodov 2009). These high fields are inferred independently of energetics from their measured spin periods $P$ and spin-down rates $\dot{P}$ under the standard assumption of magnetic dipole braking in a vacuum. With the currently known objects numbering only two dozens, ${ }^{11}$ and with this group exhibiting a wide variety of interesting, often dramatic phenomena (Woods \& Thompson 2006; Mereghetti 2013), the physics of magnetars is still poorly understood. Each new magnetar potentially provides another important piece of the neutron star puzzle.

On 2013 April 24 UT, Swift monitoring of the Sgr A* region revealed a large X-ray flare (Degenaar et al. 2013). On April 26 , the Swift/BAT instrument detected a short ( $32 \mathrm{~ms})$ X-ray

\footnotetext{
11 See the complete online magnetar catalog at

http://www.physics.mcgill.ca/ pulsar/magnetar/main.html.
}

burst consistent with that from a magnetar. This event triggered an immediate follow-up using Swift's X-Ray Telescope (XRT) that localized a new point source at a position consistent with that of Sgr A* (Kennea et al. 2013b). NuSTAR initiated a targetof-opportunity (ToO) observation on April 26 that revealed 3.76 s pulsations for the new source (Mori et al. 2013). The combination of a magnetar-like burst, periodicity, and spectrum led to the identification of the transient as a likely new magnetar in outburst. A Chandra/HRC-S observation made on April 29 confirmed the pulsations and localized the pulsar to be $\sim 3^{\prime \prime}$ from the position of Sgr A* (Rea et al. 2013). Radio observations also detected pulsations at the X-ray period (Eatough et al. 2013b), implying a dispersion measure range consistent with a position at the Galactic center (Eatough et al. 2013a, 2013c; Lee et al. 2013), while the $N_{\mathrm{H}}$ measured in the X-ray band is consistent with a position at the Galactic center or slightly beyond.

In this Letter, we detail the NuSTAR discovery of this new magnetar and a Swift observation obtained 7.3 days later that provides a confirming spin-down measurement. In Section 2 we describe the NuSTAR observations, in Section 3 we present the pulsar discovery, and in Section 4 we present the spectral analysis. Finally, in Section 5 we discuss the implications of 

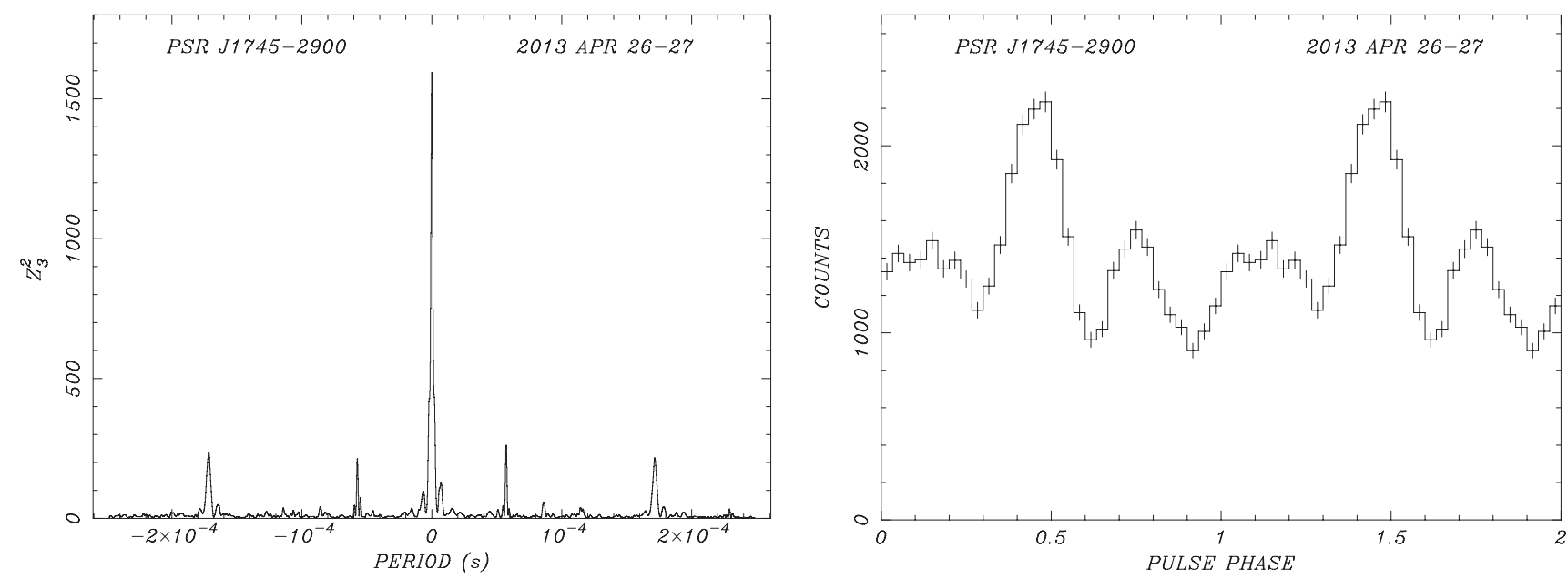

Figure 1. Discovery observation of X-ray pulsations from SGR J1745-29 using data collected from a $94.5 \mathrm{ks} N u S T A R$ observation of Sgr A*. Left: the $Z_{3}^{2}$-statistic periodogram shows a highly significant detection around $P=3.7635 \mathrm{~s}$. Side-lobe aliases from the satellite orbit are evident. Right: the light curve folded on the peak period of the periodogram. Two cycles are shown for clarity. The phase offset is arbitrary.

a magnetar close to the Galactic center. A companion paper focuses on the detection of the discovery of the SGR burst and monitoring its flux evolution (Kennea et al. 2013a).

\section{NUSTAR OBSERVATIONS}

Following the Swift/BAT report of flaring activity from the Galactic center (Degenaar et al. 2013), NuSTAR initiated a ToO observation on 2013 April 26, 1:17:31 UT. This observation had $94.5 \mathrm{ks}$ of exposure. A second observation was carried out on May 4 at UT 17:49:21 for $42.0 \mathrm{ks}$. In both observations, the Galactic center region was imaged with the two co-aligned X-ray telescopes on board NuSTAR with the Sgr A* source placed at the optical axis. These mirror/detector units provide 58" (half-power diameter) and 18" (FWHM) imaging resolution over the 3-79 keV X-ray band, with a characteristic spectral resolution of $400 \mathrm{eV}$ (FWHM) at $10 \mathrm{keV}$ (Harrison et al. 2013).

The nominal reconstructed $N u S T A R$ coordinates are accurate to $7 " .5$ (90\% confidence level). Time tagged photon arrival times are accurate to $<2 \mu \mathrm{s}$; the precise time resolution depends on the count rate incident on the detector. For the observations reported here, dead time is unimportant. The absolute timing accuracy of the NUSTAR time stamps is limited to $\sim 2 \mathrm{~ms} \mathrm{rms}$ after calibrating the thermal drift of the on-board clock.

Data were reduced and analyzed using the NuSTAR Data Analysis Software (NuSTARDAS) v10.1 in conjunction with FTOOLS 6.13. The data were filtered for intervals of high background. Photon arrival times were corrected to the solar system barycenter using the Chandra coordinates reported by Rea et al. (2013). Examination of the count rate in the NuSTAR image at the Chandra reported coordinates of the burst shows clear evidence for enhanced X-ray emission in the region. Data extracted from a $1^{\prime}$ radius aperture in the $3-10 \mathrm{keV}$ and $10-79 \mathrm{keV}$ bands yield count rates of $0.730 \pm 0.002$ counts $\mathrm{s}^{-1}$ and $0.099 \pm 0.002$ counts $\mathrm{s}^{-1}$, respectively, 2.6 and 1.2 times higher than those of the pre-flare observations. The pre-flare background rates were established using three recent NuSTAR Galactic survey observations acquired in 2012 July, August, and October.

\section{TIMING ANALYSIS}

To search for pulsations, we used an initial $7 \mathrm{ks}$ of data acquired in the first ToO pointing. A total of 16,500 photon arrival times were extracted in the full energy bandpass using a $30^{\prime \prime}$ radius aperture centered on the burst location. The arrival times were binned at $2 \mathrm{~ms}$ and searched for coherent pulsations up to the Nyquist frequency using a $2^{22}$ fast Fourier transform. We found a complex signal with three highly significant Fourier components at $1.25 \mathrm{~s}, 3.76 \mathrm{~s}, 1.88 \mathrm{~s}$, and $0.940 \mathrm{~s}$, ordered by decreasing strength.

We then carried out a refined $Z_{\mathrm{n}}^{2}$ analysis using the entire $94.5 \mathrm{ks}$, restricting the energy band to $3-10 \mathrm{keV}$ above which the source photons are dominated by the quiescent background. This allowed us to identify the fundamental at $3.76 \mathrm{~s}$ with power at odd harmonics. This signal corresponds to a pulse profile with three resolved peaks each $0.6 \mathrm{~s}$ wide, dominated by a single strong peak (see Figure 1). A $Z_{3}^{2}$ analysis yields a period $P=3.76354455(71) \mathrm{s}$ at Epoch MJD(TDB) 56409.2657 where the $1 \sigma$ error on the least significant digits is given in parentheses. The uncertainty is estimated from a Monte Carlo simulation of the light curve using the method described by Gotthelf et al. (1999).

The intrinsic pulsed fraction in the $3-10 \mathrm{keV}$ band is $f_{p}=$ $27 \% \pm 3 \%$ after allowing for the background level in the source aperture, estimated using data from the earlier observations. Here, we define the pulse fraction as the ratio of the pulsed emission to the source (background-subtracted) flux. To determine the unpulsed level, because of the high counting statistics, we take the lowest bin in the well-resolved 30-bin folded light curve shown in Figure 1. The pulse shape shows little change with energy below $7 \mathrm{keV}$, within statistics. Above $7 \mathrm{keV}$, the main pulse sharpens and the smaller sub-pulses become lost in the increasing background counts.

Following the NuSTAR detection of SGR J1745-29, we requested a Swift ToO observation to monitor its temporal and spectral evolution. A total of $15.5 \mathrm{ks}$ of X-ray data were collected on 2013 May 3 starting at UT 10:02:43.72 using the Swift/XRT (Burrows et al. 2005) in windowed timing mode. In this mode, the XRT is sensitive to photons in the $0.2-10 \mathrm{keV}$ band with $1.77 \mathrm{~ms}$ time resolution. The XRT quick-look data were processed with xrtpipeline (ver. 0.12.6) and photon arrival times were corrected to the barycenter using the Chandra coordinates. From a total of 4900 counts collected from the source in a 0.4 radius aperture in the $0.3-10 \mathrm{keV}$ XRT bandpass we detect the pulsar signal with high significance and measure a period of 3.7635603(68) s at epoch MJD(TDB) 56415.4186. A 
Table 1

Spectral Modeling of the Swift and NUSTAR Data

\begin{tabular}{lcc}
\hline \hline Model & BB & BB+PL \\
\hline$N_{\mathrm{H}}\left(10^{22} \mathrm{~cm}^{-2}\right)$ & $12.98_{-0.52}^{+0.54}$ & $14.20_{-0.65}^{+0.71}$ \\
$k T(\mathrm{keV})$ & $1.000 \pm 0.010$ & $0.956_{-0.017}^{+0.015}$ \\
BB flux $\left(\mathrm{erg} \mathrm{cm}^{-2} \mathrm{~s}^{-1}\right)$ & $(4.39 \pm 0.04) \times 10^{-11}$ & $(4.73 \pm 0.04) \times 10^{-11}$ \\
BB luminosity $\left(\mathrm{erg} \mathrm{s}^{-1}\right)$ & $\ldots$ & $(3.62 \pm 0.03) \times 10^{35}$ \\
BB radius $(\mathrm{km})$ & $\ldots$ & $1.7 \pm 0.1$ \\
$\Gamma$ & $\ldots$ & $1.47_{-0.37}^{+0.46}$ \\
PL flux $\left(\mathrm{erg} \mathrm{cm}^{-2} \mathrm{~s}^{-1}\right)$ & $\ldots$ & $(6.22 \pm 0.57) \times 10^{-12}$ \\
$\chi_{\mathrm{r}}^{2}($ dof $)$ & $1.44(466)$ & $1.01(464)$ \\
\hline
\end{tabular}

Notes. $N_{\mathrm{H}}$ is the column density, $k T$ is the temperature of the blackbody, and $\Gamma$ is the photon index of the power law. The $2-79 \mathrm{keV}$ fluxes are given for the individual components. The goodness of fit is evaluated by the reduced $\chi^{2}$ and the degrees of freedom are given between brackets. The errors are $90 \%$ confidence $\left(\Delta \chi^{2}=2.7\right)$. The blackbody radius is assuming a distance of $8 \mathrm{kpc}$.

preliminary period derivative obtained by combining this value with the above NUSTAR measurement was reported in Gotthelf et al. (2013).

Adding the two $N u S T A R$ pointings and the $S$ wift data together, we searched over $(f, \dot{f})$-space around the initial values reported in Gotthelf et al. (2013) using the $Z_{3}^{2}$ statistic to derive a revised spin-down rate of $\dot{P}=(6.5 \pm 1.4) \times 10^{-12}$, which, taken at face value, implies a magnetic field $B=1.6 \times 10^{14} \mathrm{G}$, spindown power $\dot{E}=5 \times 10^{33} \mathrm{erg} \mathrm{s}^{-1}$, and characteristic age $P / 2 \dot{P}=9 \mathrm{kyr}$, assuming a vacuum dipole. A similar $\dot{P}$ value $\left(6.5 \times 10^{-12}\right)$ was measured by a follow-up radio observation of SGR J1745-29 (Eatough et al. 2013a). $\dot{E}$ is smaller than the concurrent X-ray luminosity by orders of magnitude, which rules out rotation power as the source of the X-ray emission. We note that the spin-down rate of magnetars can be highly variable, especially following outburst (Kaspi et al. 2003; Dib et al. 2008).

We also searched the NUSTAR 3-79 keV light curves over a range of timescales for magnetar-like bursts similar to the $32 \mathrm{~ms}$ burst reported in Kennea et al. (2013b). A burst with the reported properties would have easily been detected in our data. However, we did not detect any statistically significant bursts.

\section{SPECTRAL ANALYSIS}

We analyzed the full spectral data from the first $\mathrm{NuS}$ $T A R$ observation, which consists of two consecutive data sets (ObsIDs 30001002006 and 80002013002 ) for a total of $94.5 \mathrm{ks}$. The extraction region of $1^{\prime}$ in radius encompasses strong diffuse emission and numerous unresolved sources within the Sgr A complex, so we extracted the background from a past observation in which SGR J1745-29 was not detected and Sgr A* did not exhibit any detectable flare (ObsID 30001002003 from 2012 August 4 at UT 07:56 to August 6 at UT 01:06).

Joint fitting with Swift was conducted to better constrain the column density. Five Swift/XRT observations that covered the first NuSTAR observation window were used (Swift Seq. Nos. 00554491001, 0009173620, 0009173621, 00554491991, and 00035650242 ), yielding $26 \mathrm{ks}$ exposure time in total. The data were reduced with xrtpipeline. A $22^{\prime \prime}$ radius aperture was used to extract source photons, and the background contribution was estimated by extracting photons from a concentric annulus of inner radius $70^{\prime \prime}$ and outer radius $160^{\prime \prime}$.

Joint spectral analysis was done in the $1.7-8.0 \mathrm{keV}$ energy band for Swift data and 3-79 keV for NuSTAR data using

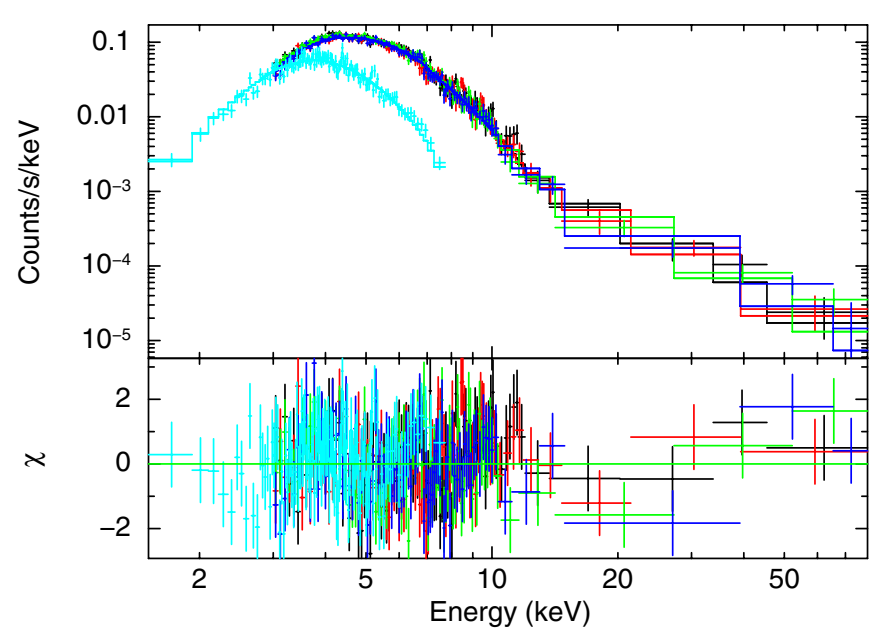

Figure 2. NUSTAR (black and red for telescope modules A and B, respectively, from ObsID 30001002006, and green and blue for telescope modules A and B, respectively, from ObsID 80002013002) and Swift (cyan) spectra jointly fitted to an absorbed blackbody plus power-law model. The crosses show the data points with $1 \sigma$ error bars and the solid lines show the best-fit model. The lower panel shows the deviation from the model in units of standard deviation.

(A color version of this figure is available in the online journal.)

XSPEC (Arnaud 1996), setting the atomic cross sections to Verner et al. (1996) and the abundances to Wilms et al. (2000). Table 1 shows the results. The low-energy spectrum is well fit by an absorbed blackbody (BB), but the high-energy tail clearly requires the addition of a power-law (PL) component. The model TBabs $\times$ (bbody + pegpwrl $w)$ yields a reduced $\chi^{2}$ of 1.01 (Figure 2). The following fluxes were extracted using the convolution model cflux for the best-fit BB + PL model over the joint energy band: the absorbed flux is $(2.67 \pm 0.02) \times 10^{-11}$ $\mathrm{erg} \mathrm{cm} \mathrm{cm}^{-2} \mathrm{~s}^{-1}$ and the unabsorbed flux is $(4.55 \pm 0.04) \times 10^{-11}$ erg cm$~^{-2} \mathrm{~s}^{-1}$. Placing the source at the Galactic center (distance of $8 \mathrm{kpc}$; Reid 1993), the inferred $2-79 \mathrm{keV}$ luminosity is $3.5 \times 10^{35}$ erg s$^{-1}$.

We also investigated any phase dependence of the NuSTAR spectrum by segmenting the data into six non-overlapping intervals, consisting of three peak and three off-peak regions. The background spectrum was identical to what was used in the phase-averaged spectral analysis detailed above, and was corrected to account for the phase cuts. A consequence of such fine division is poor photon statistics above $\sim 10 \mathrm{keV}$. Accordingly, the phase-resolved spectra were only able to constrain an absorbed BB model with a fixed column density. The phase-resolved spectra were fit with an absorbed BB model holding $N_{\mathrm{H}}$ fixed to $13 \times 10^{22} \mathrm{~cm}^{-2}$. We found a $4 \% \pm 2 \%$ variation in $k T$ while the BB flux normalization varied by $\sim 30 \%$.

\section{DISCUSSION}

The Swift-observed magnetar-like burst, the detected spin period $(3.76 \mathrm{~s})$, and the preliminary first derivative as measured by NuSTAR and Swift provide firm evidence that SGR J1745-29 is a new magnetar in outburst. SGR J1745-29 has shown no significant flux variation over $\sim 10$ days since the burst was detected on 2013 April 24 (Kennea et al. 2013a).

In archival data, there is no X-ray counterpart at the Chandra position of SGR J1745-29. This sets an upper limit on the quiescent $2-10 \mathrm{keV}$ luminosity of $\lesssim 10^{32} \mathrm{erg} \mathrm{s}^{-1}$ (Muno et al. 2009) while the bursting 2-10 keV luminosity of SGR J1745-29 reached $\sim 3 \times 10^{35} \mathrm{erg} \mathrm{s}^{-1}$. Comparable dynamic ranges have 
been seen in other magnetars, including 1E 1547-5408 (Scholz \& Kaspi 2011) and Swift J1822.3-1606 (e.g., Scholz et al. 2012); such sources have been dubbed "transient" (e.g., Halpern et al. 2008) to distinguish them from the "classical" magnetars with high quiescent luminosities (see Kaspi \& Boydstun 2010 and references therein). With its detection at radio wavelengths (Eatough et al. 2013b, 2013c), SGR J1745-29 is similar to the transient magnetars 1E 1547-5408 (Camilo et al. 2007), J1622-4950 (Levin et al. 2010), and XTE J1810-197 (Camilo et al. 2006), the only three radio-detected magnetars. That all radio-detected magnetars are transients in spite of deep radio searches of classical magnetars (Burgay et al. 2006; Crawford et al. 2007; Lazarus et al. 2012) suggests that the radio emission may be associated only with the transients. The lack of a quiescent Chandra counterpart indicates that SGR J1745-29 has a quiescent temperature of $k T \lesssim 0.3 \mathrm{keV}$, assuming that the source is at the Galactic center ( $8 \mathrm{kpc})$. This is comparable to the quiescent temperature of the transient magnetar XTE J1810-197 (Gotthelf et al. 2004) and, interestingly, to the BB temperatures of a growing number of high-magnetic-field radio pulsars (Olausen et al. 2013). This fact, and the detection of a magnetar-like outburst from one high-B pulsar (Gavriil et al. 2008), suggests that all high-B radio pulsars may be magnetars in quiescence, consistent with models of magnetothermal evolution (e.g., Pons \& Perna 2011; Perna \& Pons 2011).

The hard X-ray PL component with slope $\Gamma \sim 1$ is similar to what has been observed for other magnetars at high energies (e.g., den Hartog et al. 2008; Enoto et al. 2010). It is suggested to be generated by an electron-positron flow in a closed twisted bundle of magnetic field lines (Beloborodov 2013). In this model, the flow is sustained through $e^{ \pm}$discharge at voltage $\Phi=10^{9}-10^{10} \mathrm{~V}$, and energy is released with rate $L=I \Phi$ where $I$ is the electric current circulating in the twisted bundle. If the bundle is near the magnetic dipole axis and emerges from a polar cap of area $A$, its magnetic flux is $A B$ (where $B$ is the surface magnetic field and $A=10^{11.5} A_{11.5} \mathrm{~cm}^{2}$ ) and it generates luminosity $L \sim 10^{35} \mu_{32} \Phi_{10}\left(A_{11.5}\right)^{2} \mathrm{erg} \mathrm{s}^{-1}$ where $\mu=\mu_{32} \times 10^{32} \mathrm{G} \mathrm{cm}^{3}$ is the magnetic dipole moment. The measured $\dot{P}$ gives $\mu \approx 1.6 \times 10^{32} \mathrm{G} \mathrm{cm}^{3}$, and one finds that the observed luminosity $L$ corresponds to $A \sim 3 \times 10^{11} \mathrm{~cm}^{2}$, which is close to the emission area of the thermal component of the SGR J1745-29 spectrum. Thus, the thermal emission can be associated with the footpoint of the twisted bundle. It is bombarded by the relativistic particles from the $e^{ \pm}$discharge, and a fraction of power $L$ released in the bundle is radiated in a quasi-thermal form at the footpoint, whose temperature may be estimated from $A \sigma T^{4} \sim L$. This gives $k T \sim 1 \mathrm{keV}$, consistent with the observed thermal component. Additional heat diffusion from the deeper crust could also contribute to the spot emission (Lyubarsky et al. 2002) although the available data do not yet require this. The expected decay time of the magnetospheric luminosity is given by $t_{\mathrm{ev}} \sim 10^{7} \mu_{32} \Phi_{10}^{-1} A_{11.5} \mathrm{~s}$ (Beloborodov 2009). SGR J1745-29 is predicted in this model to show a decay time of $t_{\mathrm{ev}} \sim 10^{7} \mathrm{~s}$, which is similar to that observed in XTE J1810-197. Additional monitoring with Swift can confirm our model prediction on the flux evolution (Kennea et al. 2013a).

In spite of the angular proximity of Sgr A* to the magnetar and the possibility that the latter is in the Galactic center, we would not expect measurable bias in the spin-down rate due to orbital acceleration unless the orbit were very eccentric and the orbital phase highly fortuitous, and/or the currently measured spin-down rate were temporarily much larger than the intrinsic value. Additionally, if at the Galactic center, the magnetar is $\sim 1^{\prime \prime} .5$ outside a disk with mean eccentricity $\sim 0.3$ (Beloborodov et al. 2006; Lu et al. 2009) of clockwise rotating, predominantly massive O-type and Wolf-Rayet (WR) stars with disk ages of $\sim 6$ Myr (Genzel et al. 2010). It would not be surprising that the magnetar would stay well localized if it were born in the disk. The disk escape velocity is $\sim 750 \mathrm{~km} \mathrm{~s}^{-1}$, much larger than measured magnetar velocities (e.g., Tendulkar et al. 2012). Moreover, depending on the precise details of initial eccentricity and orbital phase, a kick velocity $\sim 100-200 \mathrm{~km} \mathrm{~s}^{-1}$ could move the magnetar out of the disk and into an even more elliptical, bound orbit. We need not, however, specify the argument to the disk. Approximately 50\% of the O/WR stars, also of ages $\sim 6 \mathrm{Myr}$, reside on extended, isotropic orbits outside the disk, and the magnetar would be similarly bound in that case. There are other stars in this region. B stars have a density 3-4 times lower than the early-type stars though. The density of much less massive late-type stars is $\sim 2$ times higher. Thus, plausibly associating the magnetar with the early-type stars, as has been previously suggested (Ritchie et al. 2010), would imply a progenitor mass $\gtrsim 40 M_{\odot}$ based on the age of all the early-type stars in this region and evolutionary models.

\section{CONCLUSION}

We report the NUSTAR discovery of a new magnetar known as SGR J1745-29. The detection of spin period and its first derivative confirms that SGR J1745-29 is a transient magnetar previously undetected. SGR J1745-29 is the fourth of a growing subclass of magnetars detected in the radio band. It indicates that many magnetars in quiescence remain undetected in the X-ray band or they have been detected as high-B radio pulsars. Further monitoring of SGR J1745-29 by X-ray and radio telescopes will reveal the time evolution of the spectral and timing properties, thus constraining the outburst emission mechanism of transient magnetars. This discovery of a magnetar near Sgr A* has important implications for the dynamics, progenitor masses, and formation of neutron stars in the vicinity of the Galactic center, and these issues will be addressed in our follow-up paper.

This work was supported under NASA Contract No. NNG08FD60C, and made use of data from the NuSTAR mission, a project led by the California Institute of Technology, managed by the Jet Propulsion Laboratory, and funded by the National Aeronautics and Space Administration. The authors thank Arash Bodaghee and Clio Sleator for their assistance with data analysis and Brian Metzger for helpful discussions.

\section{REFERENCES}

Arnaud, K. A. 1996, in ASP Conf. Ser. 101, Astronomical Data Analysis Software and Systems V, ed. G. H. Jacoby \& J. Barnes (San Francisco, CA: ASP), 17

Beloborodov, A. M. 2009, ApJ, 703, 1044

Beloborodov, A. M. 2013, ApJ, 762, 13

Beloborodov, A. M., Levin, Y., Eisenhauer, F., et al. 2006, ApJ, 648, 405

Burgay, M., Rea, N., Israel, G. L., et al. 2006, MNRAS, 372, 410

Burrows, D. N., Hill, J. E., Nousek, J. A., et al. 2005, SSRv, 120, 165

Camilo, F., Ransom, S. M., Halpern, J. P., \& Reynolds, J. 2007, ApJL, 666, L93

Camilo, F., Ransom, S. M., Halpern, J. P., et al. 2006, Natur, 442, 892

Crawford, F., Hessels, J. W. T., \& Kaspi, V. M. 2007, ApJ, 662, 1183

Degenaar, N., Reynolds, M., Miller, J., Kennea, J., \& Wijnands, R. 2013, ATel, 5006

den Hartog, P. R., Kuiper, L., Hermsen, W., et al. 2008, A\&A, 489, 245

Dib, R., Kaspi, V. M., \& Gavriil, F. P. 2008, ApJ, 673, 1044

Eatough, R., Karuppusamy, R., Champion, D., et al. 2013a, ATel, 5058

Eatough, R., Karuppusamy, R., Kramer, M., et al. 2013b, ATel, 5040

Eatough, R., Karuppusamy, R., Kramer, M., et al. 2013c, ATel, 5043 
Enoto, T., Nakazawa, K., Makishima, K., Nakagawa, Y. E., \& Sakamoto, T. 2010, PASJ, 62, 475

Gavriil, F. P., Gonzalez, M. E., Gotthelf, E. V., et al. 2008, Sci, 319, 1802

Genzel, R., Eisenhauer, F., \& Gillessen, S. 2010, RvMP, 82, 3121

Gotthelf, E., Mori, K., Halpern, J., et al. 2013, ATel, 5046

Gotthelf, E. V., Halpern, J. P., Buxton, M., \& Bailyn, C. 2004, ApJ, 605,368

Gotthelf, E. V., Vasisht, G., \& Dotani, T. 1999, ApJL, 522, L49

Halpern, J. P., Gotthelf, E. V., Reynolds, J., Ransom, S. M., \& Camilo, F. 2008, ApJ, 676, 1178

Harrison, F. A., Craig, W. W., Christensen, F. E., et al. 2013, ApJ, 770, 103

Kaspi, V. M., \& Boydstun, K. 2010, ApJL, 710, L115

Kaspi, V. M., Gavriil, F. P., Woods, P. M., et al. 2003, ApJL, 588, L93

Kennea, J., Burrows, D. N., Kouveliotou, C., et al. 2013a, ApJL, 770, L24

Kennea, J., Krimm, H., Barthelmy, S., et al. 2013b, ATel, 5009

Lazarus, P., Kaspi, V. M., Champion, D. J., Hessels, J. W. T., \& Dib, R. 2012, ApJ, 744,97

Lee, K., Eatough, R., Karuppusamy, R., et al. 2013, ATel, 5064

Levin, L., Bailes, M., Bates, S., et al. 2010, ApJL, 721, L33

Lu, J. R., Ghez, A. M., Hornstein, S. D., et al. 2009, ApJ, 690, 1463

Lyubarsky, Y., Eichler, D., \& Thompson, C. 2002, ApJL, 580, L69
Mereghetti, S. 2013, in Proc. 26th Texas Symp. on Relativistic Astrophysics (arXiv:1304.4825)

Mori, K., Gotthelf, E., Barriere, N., et al. 2013, ATel, 5020

Muno, M. P., Bauer, F. E., Baganoff, F. K., et al. 2009, ApJS, 181, 110

Olausen, S. A., Zhu, W. W., Vogel, J. K., et al. 2013, ApJ, 764, 1

Perna, R., \& Pons, J. A. 2011, ApJL, 727, L51

Pons, J. A., \& Perna, R. 2011, ApJ, 741, 123

Rea, N., Esposito, P., Israel, G., et al. 2013, ATel, 5032

Reid, M. J. 1993, ARA\&A, 31, 345

Ritchie, B. W., Clark, J. S., Negueruela, I., \& Langer, N. 2010, A\&A, 520, A48

Scholz, P., \& Kaspi, V. M. 2011, ApJ, 739, 94

Scholz, P., Ng, C.-Y., Livingstone, M. A., et al. 2012, ApJ, 761, 66

Tendulkar, S. P., Cameron, P. B., \& Kulkarni, S. R. 2012, ApJ, 761, 76

Thompson, C., \& Duncan, R. C. 1995, MNRAS, 275, 255

Thompson, C., \& Duncan, R. C. 1996, ApJ, 473, 322

Thompson, C., Lyutikov, M., \& Kulkarni, S. R. 2002, ApJ, 574, 332

Verner, D. A., Ferland, G. J., Korista, K. T., \& Yakovlev, D. G. 1996, ApJ, 465,487

Wilms, J., Allen, A., \& McCray, R. 2000, ApJ, 542, 914

Woods, P. M., \& Thompson, C. 2006, in Soft Gamma Repeaters and Anomalous X-ray Pulsars: Magnetar Candidates, ed. W. H. G. Lewin \& M. van der Klis (Cambridge: Cambridge Univ. Press), 547 